\title{
Spectrum of Rudimentary Horn Pregnancy: A Case Series
}

\author{
Prashanthi Selvaraj ${ }^{1}$, Moushmi Parpillewar ${ }^{2} \odot$ Juzar Fidvi $^{3}$
}

\begin{abstract}
Unicornuate uterus occurs due to incomplete formation and fusion of the bilateral mullerian ducts. Pregnancy in this incompletely formed horn i.e., rudimentary horn pregnancy (RHP), can present with a wide range of symptoms that may be similar to ectopic pregnancies or may remain silent like a normal pregnancy. We present a case series of three women diagnosed with RHP in different periods of gestation at a tertiary care hospital in Central India. The need for high suspicion and the role of ultrasound in the accurate diagnosis of this obstetric emergency is highlighted. The literature is well stocked with case reports on RHP with different outcomes. These three cases are being reported because of their rarity. Keywords: Rudimentary horn pregnancy, Rupture, Secondary abdominal pregnancy, Unicornuate uterus. Journal of South Asian Federation of Obstetrics and Gynaecology (2021): 10.5005/jp-journals-10006-1852
\end{abstract}

\section{BACKGROUND}

Unicornuate uterus with rudimentary horn is a mullerian duct malformation. The true incidence of mullerian duct anomalies a difficult to assess. Reported prevalence ranges from 0.16 to $10 \%$. The prevalence in women with subfertility is $1 \%$ and in women with repeated pregnancy loss approximates $3 \% .{ }^{1}$ Rudimentary horn pregnancy (RPH) is a rare obstetric event with incidence less than 1 in 70,000 to $1,50,000$ pregnancies. $^{2}$ The outcome of such pregnancies is diverse, from increased rate of miscarriage, recurrent pregnancy loss, rupture of the rudimentary horn leading to hemoperitoneum, and preterm labour. The conception can be explained either by the transperitoneal migration of sperms or the ovum or by the microconnecting passage present within the fibrous band between the rudimentary horn and the uterine cavity. The presence of a functional cavity in the contralateral part is the only clinically important factor for complications, such as hematometra or ectopic pregnancy, and treatment (removal) is always recommended even if the horn is communicating. The associated renal anomalies are reported to be as high as 50 to $80 \% .{ }^{2}$ Evaluation of the renal system is mandatory in all cases either by MRI or intravenous urogram.

\section{Case Description}

\section{Case 1}

Prerupture Diagnosis of RHP with Laparoscopic Resection of the Noncommunicating Rudimentary Horn

A 30-year-old $G_{3} P_{1} D_{1} A_{1}$ was referred from a subdistrict hospital to our obstetric casualty with a referral letter stating uterine anomaly with the missed abortion of $8+2$ weeks gestation with patent ductus arteriosus (PDA). She had history of amenorrhea for 2 months with complaints of bleeding per vaginum on and off since 5 days and lower abdominal pain since 2 days. She was married sin ce 7 years. All her previous conceptions were spontaneous. She had a full-term cesarean section before 4 years for severe preeclampsia with abruptio placentae. Intraoperative notes from discharge card suggested unicornuate uterus without rudimentary horn, followed by a spontaneous abortion at 2 months before 2 years. The patient has been regularly following up with a cardiologist and on regular medications for PDA ( $2 \mathrm{~mm}$ with $\mathrm{L}$ to $R$ shunt). No other significant medical history. On admission, her vitals were

\footnotetext{
${ }^{1-3}$ Department of Obstetrics and Gynaecology, Government Medical College, Nagpur, Maharashtra, India

Corresponding Author: Prashanthi Selvaraj, Department of Obstetrics and Gynaecology, Government Medical College, Nagpur, Maharashtra, India, Phone: +91 9403397882, e-mail: swaha_dundoo@yahoo.com

How to cite this article: Prashanthi S, Parpillewar M, Fidvi J. Spectrum of Rudimentary Horn Pregnancy: A Case Series. J South Asian Feder Obst Gynae 2021;13(1):61-65.

Source of support: Nil

Conflict of interest: None
}

stable. On per abdominal examination $4 \times 4 \mathrm{~cm}$ vague tender mass palpable in the right iliac fossa. On per speculum examination, altered color brownish discharge seen coming through os, single cervix is seen. Bimanual examination revealed soft cervix, uterus corresponding to 8 weeks gestation deviated more toward the left and $4 \times 4 \mathrm{~cm}$ right adnexal mass present. Cervical excitation present. Her investigation revealed $\mathrm{Hb} 10.3 \mathrm{~g} / \mathrm{dL}$ and the rest of the blood counts, urea and creatinine were within normal limits. USG reveals uterus $9 \times 4.1 \times 5.8 \mathrm{~cm}$ with no gestational sac in the cavity or the cervical canal. A complex mass of $6 \times 5.5 \mathrm{~cm}$ separate from the right ovary with increased vascularity was present suggesting an ectopic pregnancy and left ovary and fallopian tube were normal. No free fluid present. The decision of laparoscopy was taken after anesthetic and cardiologist fitness. Intraoperative findings were as follows:

- Omentum found adhered to anterior abdominal wall, adhesiolysis done.

- Bulky unicornuate uterus.

- Left ovary with fallopian tube normal.

- Right-sided noncommunicating RHP (Fig. 1A) attached to the lateral pelvic wall through a small band of fibrous tissue extending from the body of the uterus (Fig. 1B).

- Right ovary normal attached to the right lateral pelvic wall.

- No hemoperitoneum.

Products of conception were suctioned through a small incision over the rudimentary horn (Fig. 1C), rest of the horn was excised and removed through the umbilical port. Postoperative recovery was uneventful.

(-) Jaypee Brothers Medical Publishers. 2021 Open Access This article is distributed under the terms of the Creative Commons Attribution 4.0 International License (https://creativecommons.org/licenses/by-nc/4.0/), which permits unrestricted use, distribution, and non-commercial reproduction in any medium, provided you give appropriate credit to the original author(s) and the source, provide a link to the Creative Commons license, and indicate if changes were made. The Creative Commons Public Domain Dedication waiver (http://creativecommons.org/publicdomain/zero/1.0/) applies to the data made available in this article, unless otherwise stated. 




Fig. 1A: Shows laparoscopic view of the enlarged noncommunicating rudimentary horn with pregnancy $(\mathrm{RH})$ alongside the unicornuate uterus $(\mathrm{U})$

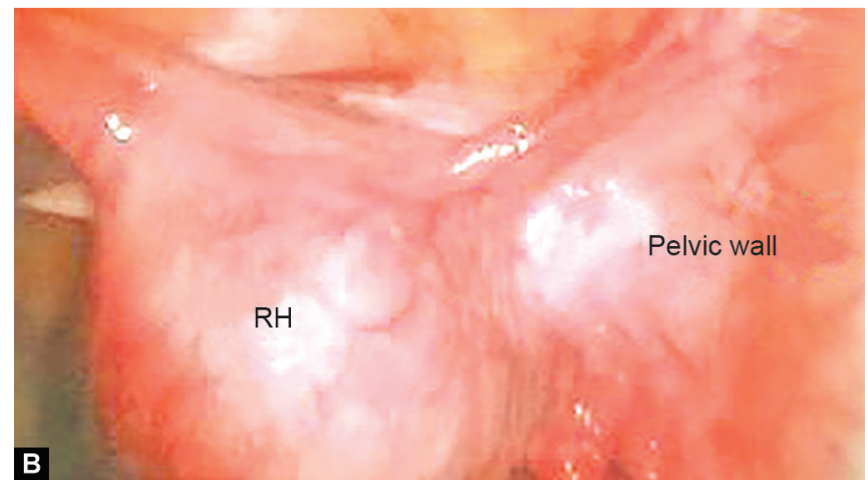

Fig. 1B: Shows the attachment of the rudimentary horn $(\mathrm{RH})$ to the lateral pelvic wall

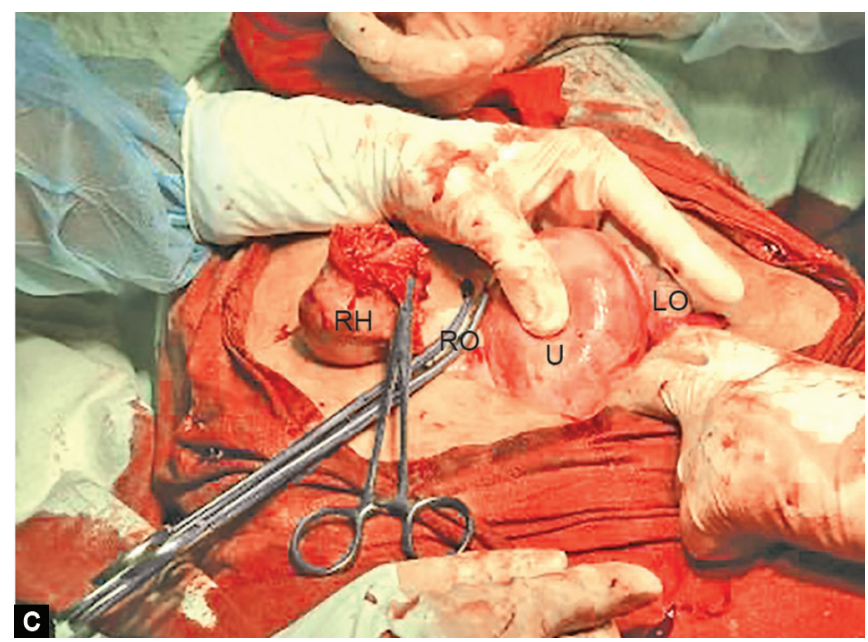

Fig. 1C: Shows the products of conception being removed through a small incision on the rudimentary horn $(\mathrm{RH})$

\section{Case 2}

\section{Ruptured Rudimentary Horn Pregnancy in Second Trimester-An Acute Obstetric Event}

A 26-year-old primigravida was referred to our emergency department from rural health center with a diagnosis of 18 weeks gestation with ruptured ectopic pregnancy of the right fallopian tube.
She had a history of vague lower abdominal pain on and off since 1 month with acute abdominal pain and three episodes of vomiting since last 12 hours and syncopal attack with shoulder tip pain since 30 minutes. No other significant medical and surgical illness. She was married since 2 years, spontaneously conceived. Urine pregnancy test done at home at 60 days of amenorrhea. Unregistered for antenatal care (ANC) with no dating scan.

On examination, she was anemic and dehydrated. Pulse rate $126 \mathrm{bpm}$, rapid feeble, systolic BP $80 \mathrm{~mm} \mathrm{Hg}$, and diastolic BP not recordable. Respiratory rate 22 /minute. On per abdominal examination, uterus size could not be made out, guarding and rigidity present. Per speculum examination reveals small blood clots through os. Bimanual examination shows fullness in fornices with cervical tenderness. Abdominopelvic ultrasound from the referral center shows a fetus corresponding to 16 weeks gestation with no cardiac activity in the right adnexa. Gross hemoperitoneum present. Diagnosis of ruptured ectopic pregnancy was made. The decision of exploratory laparotomy was taken after resuscitation. Intraoperative findings are as follows:

- Unicornuate uterus

- Left ovary and fallopian tube normal

- Right ovary appears normal, attached to lateral pelvic wall

- Ruptured right-sided communicating RHP (Fig. 2A)

- Fetus (140 g) with placental tissue present free in peritoneal cavity (Fig. 2B)

- Hemoperitoneum of $1500 \mathrm{~mL}$ present

Rudimentary horn excised.

Her postoperative recovery was good. Patient discharged home on postoperative day 7. Patient counseled regarding contraception and the need for early ANC and elective cesarean section in forthcoming pregnancies.

\section{Case 3}

Ruptured Rudimentary Horn Pregnancy with Secondary Abdominal Pregnancy

A 29-year-old $G_{2} P_{1} L_{1}$ with 8 months amenorrhea was seen in our obstetric casualty with a referral from suburban health center with renal colic. She came with complaints of acute lower abdominal pain radiating to the right loin since 2 days with burning and increased

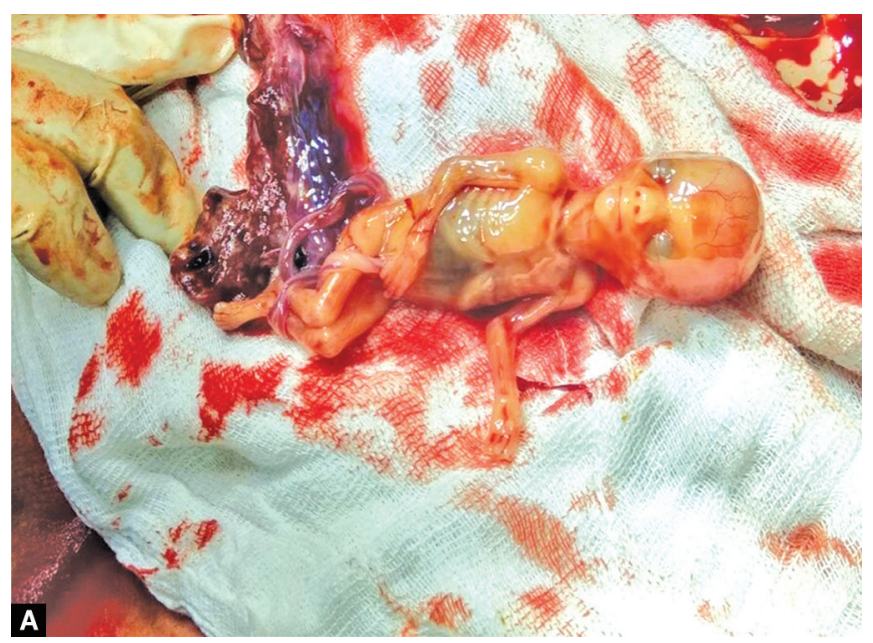

Fig. 2A: Shows the ruptured rudimentary horn $(\mathrm{RH})$ alongside the intact unicornuate uterus $(\mathrm{U})$ with normal right ovary (RO) and left ovary (LO) 


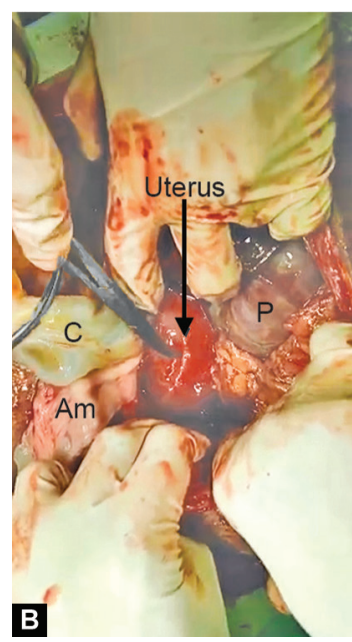

Fig. 2B: Shows the dead fetus of 14 to 16 weeks along with the placenta

frequency of micturition. She also had complaints of constipation on and off. She had no complaints of bleeding per vaginum or leaking. Rest of her antenatal period was uneventful except for the painful fetal movements. She had a full-term cesarean section before 3 years for meconium-stained liquor with fetal distress in the first stage of labor. Her antenatal and puerperal periods were uneventful. No discharge notes available with the patient. In the present pregnancy, she was booked case at a suburban district hospital with six to seven antenatal visits. She gave a history of admission at the same hospital at 3 months gestation with acute abdominal pain and vomiting. Her complaints were relieved and her condition improved with symptomatic treatment.

She had multiple ANC USG reports from different ultrasound centers. Her first antenatal ultrasound was done at $14+5$ weeks gestation suggestive of bicornuate unicollis uterus with pregnancy in the right horn and left horn empty. Placenta found developing posterior in the upper segment. Following that, she had done her second ultrasound at $27+3$ weeks gestation suggestive of bicornuate uterus with globular placenta covering internal os. AFI-18 cm. Her third ultrasound report suggested single live intrauterine gestation in changing presentation with placenta covering internal os with AFI $7 \mathrm{~cm}$.

On examination, she was moderately nourished. On abdominal examination, uterus corresponding to 34 weeks gestation. Fetus in an oblique lie with head on maternal right. Uterine contour not well maintained. Fetal parts were easily palpable. FHS $138 \mathrm{bpm}$ heard with hand Doppler.

The patient was advised admission, her complete blood count and MSU were done. Blood count was within normal limits and MSU showed gram-negative bacilli. Urine alkalinizer and antibiotics were given. The patient felt better after 48 hours of admission. Routine antenatal growth scan was done, suggested single live fetus corresponding to 33 weeks gestation in transverse lie (head towards maternal left), placenta present over the anterior wall with e/o multiple hypoechoic islands within, with loss of hypoechoic zone between the uterus and bladder s/o placenta accreta.

On day 5 of admission, the patient had complaints of reduced fetal movements since morning. Nonstress test suggested suspicious trace. Ultrasound by senior obstetrician done for $\mathrm{AFI}-2 \mathrm{~cm}$ with placenta seen in the upper segment not related to the internal os. Decision of emergency cesarean section was

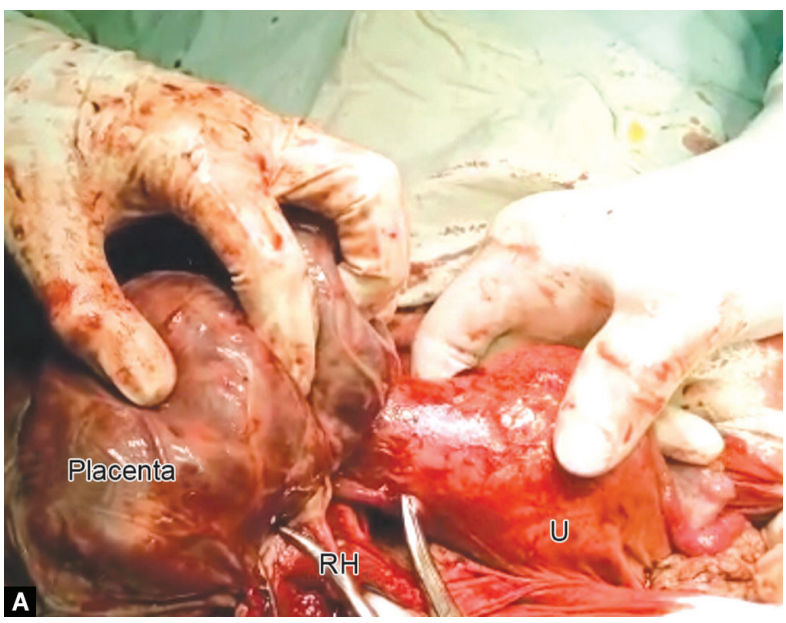

Fig. 3A: Shows the intact unicornuate uterus with the placenta $(P)$, cord (C), and amniotic membrane (AM) alongside the omentum in the abdominal cavity after delivery of a viable near term baby suggesting an abdominal pregnancy

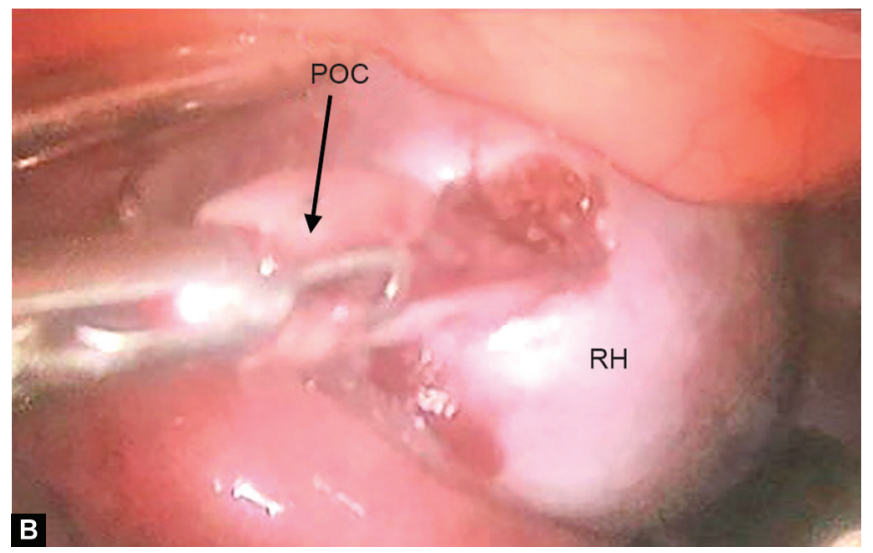

Fig. 3B: Shows the hyperplacentosis and its attachment to the rudimentary horn $(\mathrm{RH})$ with intact unicornuate uterus $(\mathrm{U})$

taken. Neonatalogist informed for the high-risk preterm neonate. Adequate blood products arranged.

Under regional anesthesia, the abdomen was opened by midline vertical incision.

Intraoperative findings are as follows:

- Single live fetus was found in transverse lie in the abdominal cavity with no amniotic cavity and liquor. Baby delivered by breech and cord clamped.

- Uterus unicornuate intact, enlarged to 12 weeks size.

- On further exploration, Placenta was found attached to the ruptured rudimentary right horn (Fig. 3A).

- Placenta was vascular and fragile with hyperplacentosis (Fig. 3B). Edges carefully palpated to determine its insertion.

- Rudimentary horn along with the placenta was removed.

- Abdomen thoroughly examined. Intestine liver and splenic surfaces were examined.

- Abdomen closed after hemostasis.

Postoperative recovery was uneventful. Healthy mother and newborn $(2.25 \mathrm{~kg})$ were discharged on the tenth postoperative day. 


\section{Discussion}

According to the European Society of Human Reproduction and Embryology (ESHRE) classification of congenital uterine anomalies, the unicornuate uterus or the hemiuterus belongs to class $4\left(U_{4}\right)$. This class incorporates all cases of unilateral formed uterus. The contralateral part could either be incompletely formed or absent. It is a formation defect rather than a fusion or absorption defect. The class $\mathrm{U}_{4}$ is further divided into two classes:

- Unicornuate uterus with a rudimentary cavity characterized by the presence of a communicating or noncommunicating functional horn.

- Unicornuate uterus without a rudimentary cavity characterized either by the presence of a nonfunctional contralateral uterine horn or aplasia of the contralateral part.

The connection of the horn with the uterus may be fibrous or fibromuscular.

\section{Case 1}

In the past, majority of the cases were diagnosed after rupture of rudimentary horn. MRI has been a useful preoperative tool for diagnosing RHP and any abnormal placentation. However, they are not readily available in all centers. A strong clinical suspicion of a functional rudimentary cavity in spite of the previous discharge notes of aplasia of contralateral part led to the laparoscopy and clenched the diagnosis of RHP in this case. The rudimentary horn during the previous LSCS could have gone unnoticed as it was attached to the lateral pelvic wall and connected to the uterus only through a small fibrous band. Prerupture diagnosis of RHP allows laparoscopic resection of the horn ideal because of the lesser blood loss and earlier postoperative recovery.

Buntugu et al. ${ }^{2}$ placed a foley's catheter into the uterine cavity prior to performing a transabdominal ultrasound for diagnosing an extrauterine pregnancy. Although not accepted as a preferred method, it is the only possible intervention to make the diagnosis of the RHP when other preoperative tools like MRI are not available.

Tsafrir et al. ${ }^{3}$ reported two cases of RHP found in the first trimester by ultrasound and confirmed with MRI. Criteria for diagnosing pregnancy in rudimentary horn are as follows:

- A pseudopattern of asymmetrical bicornuate uterus

- Absent visual continuity tissue surrounding the gestation sac and uterine cervix

- Presence of myometrial tissue surrounding the gestational sac

Nonetheless, most of the cases remain a sticky wicket to diagnose.

\section{Case 2}

This case shows us the importance of a dating scan in the first trimester. Fedele et al. ${ }^{4}$ have found ultrasound to be useful in the diagnosis. Although the sensitivity of ultrasound to diagnose RHP is only $26 \%$ in the first trimester and sensitivity decreases as pregnancy advances. ${ }^{5}$ According to Shah and Khan, ${ }^{6}$ ectopic pregnancies must be suspected in every pregnant woman with pain abdomen unless proven otherwise. It is important to know that ectopic pregnancy is present commonly between sixth and tenth weeks of gestation, whereas RHP may present late, ${ }^{7}$ as in our case. Therefore, rare causes of acute abdomen in obstetrics such as RPH must be considered in a patient presenting after 10 weeks of gestation. In our case, the history of amenorrhea with a positive urine pregnancy test ruled out the other differentials. Lack of previous ultrasound reports led us to manage the patient solely based on her history, clinical examination, and laboratory parameters and do a lifesaving emergency laparotomy.

\section{Case 3}

Abdominal pregnancy is a rare obstetric event but presents with grave risk to pregnant women. Early abdominal pregnancy is self-limited but an advanced abdominal pregnancy has high fetal and maternal morbidity and mortality. The incidence of advanced abdominal pregnancy was seen in 1 in 25,000 pregnancies. ${ }^{8}$ This is a rare case of late preterm viable secondary abdominal pregnancy following rupture of a RHP in early gestation. This condition requires a high index of suspicion, as history and clinical examination are often inconclusive. She had a history of admission at rural hospital at 14 weeks for acute abdomen, probably due to rupture of the rudimentary horn seeding the pregnancy into the peritoneal cavity. The following features must alert the sonologist/obstetrician regarding abdominal pregnancy: ${ }^{9}$

- Abnormal relationship among the fetus, placenta, amniotic fluid, and uterus.

- Fetal malpresentation.

- Fetal skull or small parts overlying the maternal spine on lateral x-ray.

The frequently changing levels of amniotic fluid with different sonographers was an ominous ultrasound finding in our case. The placenta lying in the peritoneal cavity near the bladder obliterating the space between the bladder wall and uterine serosa appeared similar to central placenta previa. Maternal deaths associated with abdominal pregnancy result from hemorrhage after inadvertent removal of the placenta. ${ }^{10}$ In our patient, the placenta was attached to the ruptured rudimentary horn, making it possible to remove the whole placenta without significant hemorrhage. Removal of the entire placenta has been recommended but if found attached to vital abdominal organs like the liver or the intestine, it is safer to leave all or part of the placenta and allow it to reabsorb slowly. However, leaving the placenta in situ has been associated with increased postoperative morbidity. ${ }^{10}$ Other methods like bipolar cautery, hemostatic matrix sealant (Floseal), vasopressin, and oxidised cellulose (Surgicel) can be used to achieve hemostasis after abdominal pregnancy is separated from an abdominal organ. ${ }^{11}$ Stepwise devascularisation or arterial embolizations are other methods to reduce placental vascularity. In three cases reported by Sandberg and Pelligra, ${ }^{12}$ the diagnosis of abdominal pregnancy was made intraoperatively as in our case.

\section{Conclusion}

The diagnosis of RHP is particularly difficult due to the rarity of this condition and understanding of the disease. RHP can present at any gestation with diverse symptoms. Most of the RHP has a poor fetal outcome. The primary management is surgical removal of the rudimentary horn to avoid risk of recurrence of rupture. Similarly, there is no single diagnostic tool to diagnose abdominal pregnancy. A high index of suspicion, early diagnosis, and time management is crucial in saving the mother's life.

\section{OrCID}

Moushmi Parpillewar ㄴ https://orcid.org/0000-0002-4060-8120 


\section{References}

1. Virkud A. Modern gynecology. 3rd ed. Mumbai: APC Publishers; 2017. p. 167 [Chapter 15].

2. Buntugu KA, Ntumy MY, Ameh EO, et al. Rudimentary horn pregnancy: pre-rupture diagnosis and management. Ghana Med J 2008;42(2):92-94. Available from: https://doi.org/10.4314/gmj. v42i2.43601.

3. Tsafrir A, Rojansky N, Sela HY, et al. Rudimentary horn pregnancy: first trimester prerupture sonographic diagnosis and confirmation by magnetic resonance imaging. J Ultrasound Med 2005;24(2):219-223. DOI: 10.7863/jum.2005.24.2.219.

4. Fedele $L$, Dorta $M$, Vercellini $P$, et al. Ultrasound on the diagnosis of subclasses of unicornuate uterus. Obstet Gynecol 1988;71(2): 274-277. Available from: https://pubmed.ncbi.nlm.nih.gov/2962027/.

5. Jayasinghe S, Rane A, Stalewski $\mathrm{H}$, et al. The presentation and early diagnosis of the rudimentary uterine horn. Obstet Gynaecol 2005;105(6):1456-1467. DOI: 10.1097/01.AOG.0000161321.94364.56.

6. Shah N, Khan NH. Ectopic pregnancy: presentation and risk factors. J Coll Physicians Surg Pak 2005;15(9):535-538. Available from: https:// pubmed.ncbi.nlm.nih.gov/2962027/.
7. Sivalingam VN, Duncan WC, KirkE, et al. Diagnosis and management of ectopic pregnancy. J Fam Plann Reprod Health Care 2011;37(4): 231-240. DOI: 10.1136/jfprhc-2011-0073.

8. Desai BR, Patted Shobhana S, Pujar Yeshita V, et al. Advanced Secondary abdominal pregnancy following rupture of rudimentary horn. J Obstet Gynecol India 2005;55(2):180. Available from: https://jogi. co.in/march_april_2005/18_cro_advanced_secondary_abdominal_ pregnancy_following.pdf.

9. Costa SD, Presley J, Bastert G. Advanced abdominal pregnancy. Obstet Gynecol Surv 1991;46(8):515-525. DOI: 10.1097/00006254-19910800000003.

10. Pratiksha G, Alka S, Reeti M. Secondary abdominal pregnancy and its associated diagnostic and operative dilemma: three case reports. J Med Rep 2009;3:7382. DOI: 10.4076/1752-1947-3-7382.

11. Nilesh A, Funlayo O. Early abdominal ectopic pregnancy: challenges, update and review of current management. Obstet Gynaecol 2014;16(3):193-198. DOI: 10.1111/tog.12109.

12. Sandberg EC, Pelligra R. The medical antigravity suit for management of surgically uncontrollable bleeding with abdominal pregnancy. Am J Obstet Gynecol 1983;146(5):519-525. DOI: 10.1016/00029378(83)90793-7. 